\title{
Comparative genomic analysis reveals genetic features related to the virulence of Bacillus cereus FORC_013
}

\author{
Hyun-Jin Koo' ${ }^{1}$, Sojin Ahn ${ }^{2,3}$, Han Young Chung ${ }^{3,4}$, Suyeon Kim³ ${ }^{3,4}$, Kwondo Kim², Sangryeol Ryu ${ }^{3,4}$, \\ Ju-Hoon Lee ${ }^{3,5}$, Sang Ho Choi $i^{3,4^{*}}$ and Heebal Kim ${ }^{1,2,3^{*}}$
}

\begin{abstract}
Background: Bacillus cereus is well known as a gastrointestinal pathogen that causes food-borne illness. In the present study, we sequenced the complete genome of B. cereus FORC_013 isolated from fried eel in South Korea. To extend our understanding of the genomic characteristics of FORC_013, we conducted a comparative analysis with the published genomes of other B. cereus strains.

Results: We fully assembled the single circular chromosome (5,418,913 bp) and one plasmid (259,749 bp); 5511 open reading frames (ORFs) and 283 ORFs were predicted for the chromosome and plasmid, respectively. Moreover, we detected that the enterotoxin (NHE, HBL, CytK) induces food-borne illness with diarrheal symptom, and that the pleiotropic regulator, along with other virulence factors, plays a role in surviving and biofilm formation. Through comparative analysis using the complete genome sequence of B. cereus FORC_013, we identified both positively selected genes related to virulence regulation and 224 strain-specific genes of FORC_013.

Conclusions: Through genome analysis of B. cereus FORC_013, we identified multiple virulence factors that may contribute to pathogenicity. These results will provide insight into further studies regarding $B$. cereus pathogenesis mechanism at the genomic level.
\end{abstract}

Keywords: Bacillus cereus, Virulence, Comparative genomics, Positive selection, Unique genes

\section{Background}

For several decades, food-borne illnesses caused by microorganisms have attracted political and media attention around the world [1], because outbreaks of these diseases have a strong association with public health problems such as hospitalizations and even deaths [2, 3]. Preventing these illnesses is challenging, involving a complicated process rather than simple conventional methods [4]. Therefore, it is essential that we elucidate the phenotypic and genotypic features

\footnotetext{
*Correspondence: choish@snu.ac.kr; heebal@snu.ac.kr

${ }^{1}$ Department of Agricultural Biotechnology and Research Institute

of Agriculture and Life Sciences, Seoul National University, Seoul, Republic

of Korea

${ }^{4}$ Department of Agricultural Biotechnology, Center for Food Safety and Toxicology, Seoul National University, Seoul, Republic of Korea Full list of author information is available at the end of the article
}

of agents that cause outbreaks. Among a variety of organisms that can cause food-borne illness, Bacillus, which is characterized by several features-a grampositive, rod-shaped, motile, spore-forming and aerobic-to-facultative-has been considered an important opportunistic pathogen and may be categorized as (1) gastrointestinal pathogens inducing emetic and diarrheal symptoms and (2) systemic and local infections associated with the respiratory tracts of immunologically compromised patients and neonates $[5,6]$. There were food poisoning outbreaks from consuming food including meat, soups, vegetable dishes, dairy products, and seafood that were contaminated with Bacillus cereus $[7,8]$. Especially, B. cereus spore, which survives within the small intestine of the host, has a connection with food-borne illness inducing diarrheal symptom $[9$, 10]. A recent study reported that enterotoxins might 
cause diarrheal food-borne illness, including hemolysin BL (HBL), non-hemolytic enterotoxin (NHE) and cytotoxin $\mathrm{K}$ (CytK) [7, 11]. Although many research efforts have focused on the food-borne diseases caused by $B$. cereus, little genomic research of this species has been conducted into the mechanism of toxicity leading to food poisoning.

In the present study, we sequenced the complete genome of B. cereus FORC_013, to better understand its pathogenesis at the genomic level, which was isolated from fried eel in South Korea. Using this sequence data, we assembled the complete genome of $B$. cereus FORC_013 and determined its genomic characteristics. Then, we conducted a comparative genome analysis of the FORC_013 with the complete sequences of 29 other $B$. cereus strains to gain more information of this strain. These results may be useful in elucidating the pathogenicity of B. cereus and its role in food poisoning.

\section{Methods}

\section{Sample collection, strain isolation, and whole genome} sequencing

Bacillus cereus FORC_013 was isolated from fried eel in South Korea and cultivated in Brain Heart Infusion (BHI; Difco, Detroit, MI, USA) medium. Genomic DNA was isolated and purified using the MoBio UltraClean Microbial DNA Isolation Kit (MoBio, Carlsbad, CA, USA) following the manufacturer's recommendations. Approximately $5 \mu \mathrm{g}$ of genomic DNA was cut into 8-12 kb fragments using the Hydroshear system (Digilab, Marlborough, MA, USA). SMRTbell libraries were prepared for each sample using the DNA Template Prep kit $2.0(3-10 \mathrm{~kb})$ for SMRT sequencing which was carried out with $\mathrm{C} 2$ chemistry on a PacBio RS II system (Pacific Bioscience, Menlo Park, CA, USA). The AMPure XP bead purification system (Beckman Coulter, Brea, CA, USA) was used to purify libraries by removing small fragments $(<1.5 \mathrm{~kb})$. An Agilent 12,000 DNA kit (Applied Biosystems, Santa Clara, CA, USA) was used to characterize the size distribution of sheared DNA templates. Sequencing primers were annealed to the templates and DNA polymerase enzyme $\mathrm{C} 2$ was added following the manufacturer's instructions. Loading the enzyme template-complexes and libraries onto 75,000 zero-mode waveguides (ZMWs) was conducted using DNA/Polymerase Binding kit P4 (Pacific Bioscience) according to the manufacturer's instructions. SMRTbell library sequencing using a 120-min sequence capture protocol with PacBio RS II to maximize read length via the DNA sequencing kit Reagent 2.0 (Pacific Bioscience). The summary of sequencing result is contained in Additional file 1.

\section{Genome assembly and annotation}

Whole genome assembly was performed using the SMRT portal system. Sequencing reads from the PacBio RS II system were assembled using the HGAP assembly-3 algorithm with curation of the genome size parameter which was set to $3 \mathrm{Mb}$ using the Compute Minimum Seed Read Length option while other parameters were set to default [12]. Sequencing errors were removed and a polishing assembly process was repeatedly performed to reduce errors, such as indels in the draft assembly using Quiver until none genomic variants were detected. The genome sequences were assembled into contigs using the PacBio RS II system. The orientation and direction of the assembled sequence was defined via Basic Local Alignment Search Tool (BLAST) and Mummer analyses [13]. BioEdit software was used to curate the polished sequence based on alignment results [14]. We used Rapid Annotation of Prokaryotic Genomes (PROKKA) to predict open reading frame (ORF) count and the rRNA and tRNA contents of B. cereus FORC_013. [15] The Rapid Annotation using Subsystem Technology (RAST) [16] was used for SEED annotation with the default settings. Cluster of Orthologous Groups (COG) annotation was conducted using WebMGA [17] and DNAPlotter [18] was employed to generate an annotation map.

\section{Genome accession numbers}

To study the comparative genomics of B. cereus strains, 29 complete genome sequences were downloaded from the NCBI database (http://www.ncbi.nlm.nih.gov/genome/ genomes/157). The accession numbers for these $29 \mathrm{~B}$. cereus sequences are CP009318.1 (03BB102), CP009641.1 (03BB108), CP009941.1 (03BB87), CP009596.1 (3a), CP015727.1 (A1), CP001177.1 (AH187), CP001283.1 (AH820), AE017194.1 (ATCC10987), AE016877.1 (ATCC14579), CP009628.1 (ATCC4342), CP001176.1 (B4264), CP001746.1 (CI), CP011153.1 (CMCCP0011), CP011151.1 (CMCCP0021), CP009300.1 (D17), CP009968.1 (E33L), CP003187.1 (F837/76), CP009369.1 (FM1), CP009686.1 (FORC_005), CP012691.1 (FORC_024), CP003747.1 (FRI-35), CP008712.1 (FT9), CP009590.1 (G9241), CP001186.1 (G9842), CP011155.1 (HN001), AP007209.1 (NC7401), CP012483.1 (NJ-W), CP000227.1 (Q1), and CP009605.1 (S2-8).

\section{Analysis of virulence factors}

To investigate the virulence factor encoding genes of FORC_013 strain, we downloaded full DNA sequences from the virulence factor database (VFDB). For virulence gene identification, we used BLASTn method against VFDB (identity $\geq 0.95$ ). 


\section{Phylogenetic and comparative genome analysis}

JSpecies software was employed to compute Average nucleotide identity (ANI) values of all 30 strains [19]. The MESTORTHO algorithm [20] was used to build an orthologous gene set for the 30 complete genomes (identity $\geq 0.95$; coverage $\geq 0.8$ ). Multiple sequence alignment of each orthologous gene was conducted using PRANK [21]. After removing the poorly aligned positions using Gblocks [22], orthologous sequences were joined into one sequence to build a phylogenetic tree. The neighbor-joining method was used to construct a tree using MEGA7 [23]. The Codeml program based on PAML4 (phylogenetic analysis by maximum likelihood) [24] was used to detect the genes which were under selective pressure by estimating $\mathrm{dN}$ (rate of non-synonymous substitution) and dS (rate of synonymous substitution) based on the branch and branch-site models. Prior to pan-genome analysis, 30 complete genome sequences of $B$. cereus were annotated using the PROKKA annotation tool [15]. After annotation, GFF files output from PROKKA were used as the input files for creating the pan-genome with Roary software [25].

\section{Quality assurance}

The 16s rRNA gene was identified from the assembled sequence using PROKKA. Pairwise distances were calculated by comparison of FORC_013 with published $B$. cereus genomes using ANI values.

\section{Results and discussion}

\section{Genome features of Bacillus Cereus FORC_013}

The B. cereus FORC_013 genome consists of a circular DNA chromosome and a single circular plasmid (Additional file 1: Table S1). The whole genome sequence comprises $5,418,913 \mathrm{bp}$ with a GC content of $35.3 \%$. The $B$. cereus FORC_013 plasmid contains 259,749 bp with a GC content of 33\% and a total of 259 predicted ORFs. The FORC_013 genome contains 5424 ORFs, 107 tRNA sequences and 42 rRNA sequences. Among the predicted ORFs, 3750 (69\%) were predicted based on annotated genes and 1674 (31\%) were hypothetical and unknown proteins (Fig. 1). Figure 2 presents the categorization of estimated functional genes based on SEED subsystem categories and COG functional categories; 3525 genes were classified into 26 SEED subsystem categories. Of these, 286 ORFs were categorized into the cell wall and capsule subsystem, which includes pathogenicity; 128 ORFs were responsible for virulence, disease and defense, which may be related to toxin production. In total, 82 ORFs were related to motility and chemotaxis due to the formation of biofilms, which affect the persistence of the pathogen. Functional annotation based on COG categorization using WebMGA identified 3537 ORFs. Excluding
ORFs that were related to the general function prediction only and function unknown categories (26.4\%), 1235 ORFs, accounting for more than one-third of the COGassigned ORFs, were classified into five major COG categories: 309 ORFs in category $\mathrm{E}$ (amino acid transport and metabolism), 283 ORFs in category K (transcription), 219 ORFs in category $\mathrm{M}$ (cell wall/membrane/envelope biogenesis), 217 ORFs in category G (carbohydrate transport and metabolism) and 207 ORFs in category J (translation, ribosomal structure and biogenesis).

\section{Virulence factors}

The 20 virulence genes of FORC_013 were identified via BLASTn method against VFDB (Table 1). The virulence factors of FORC_013 were classified into six categories: host immune evasion, lipase, protease, regulation, toxin, and others. As previous studies reported, the diarrheal symptom is well known for having a close relationship with the enterotoxin, such as hemolytic enterotoxin HBL, non-hemolytic enterotoxin NHE and cytotoxin $\mathrm{K}[7,11]$. The genome of FORC_013 has all of these enterotoxins; $C y t \mathrm{~K}$ gene, $\mathrm{HBL}$ gene cluster $(h b l \mathrm{~A}, h b l \mathrm{~B}, h b l \mathrm{C}$, and $h b l \mathrm{D})$ and NHE gene cluster (nhe $\mathrm{A}$, nhe $\mathrm{B}$, and nheC), suggesting that these genes are responsible for pathogenicity of FORC_013. In the protease category, immune inhibitor A metalloprotease (inhA) was detected; this gene assists in surviving the macrophage environment, which is an important factor of the host immune system [26]. Further, this supports that inhA in FORC_013 may contribute to retain living in the macrophage intracellular system. The FORC_013 strain has hemolysin II ( $h y l$ II) and hemolysin III ( $h y l$ III) that form the pores by adapting under the harsh environment [27, 28]. We also identified an regulation protein, pleiotropic regulator $(P l c \mathrm{R})$, which is a well-known pleiotropic regulator of genes related to pathogenicity [29]. This gene plays a role in the biofilm formation, which may induce the sporulation of bacteria [30, 31]. Biofilm formation facilitates generating adhesive spores and contributes to high resistance [32]. Detection of PlcR indicated that the FORC_013 may take advantage of both biofilm formation and virulence gene regulation. Based on the results, it is reasonable to assume that these virulence factors contribute to pathogenicity of FORC_013. Additionally, we conducted a lactate dehydrogenase (LDH) release assay to identify cytotoxicity, which indicated that FORC_013 has pathogenic activity (Additional file 1: Fig S1).

\section{Phylogenetic and comparative genome analysis}

An ANI tree and a phylogenetic dendrogram based on orthologous genes were built for a comparative analysis of the FORC_013 strain. Both trees were generated using 29 complete genome sequences acquired from the NCBI 


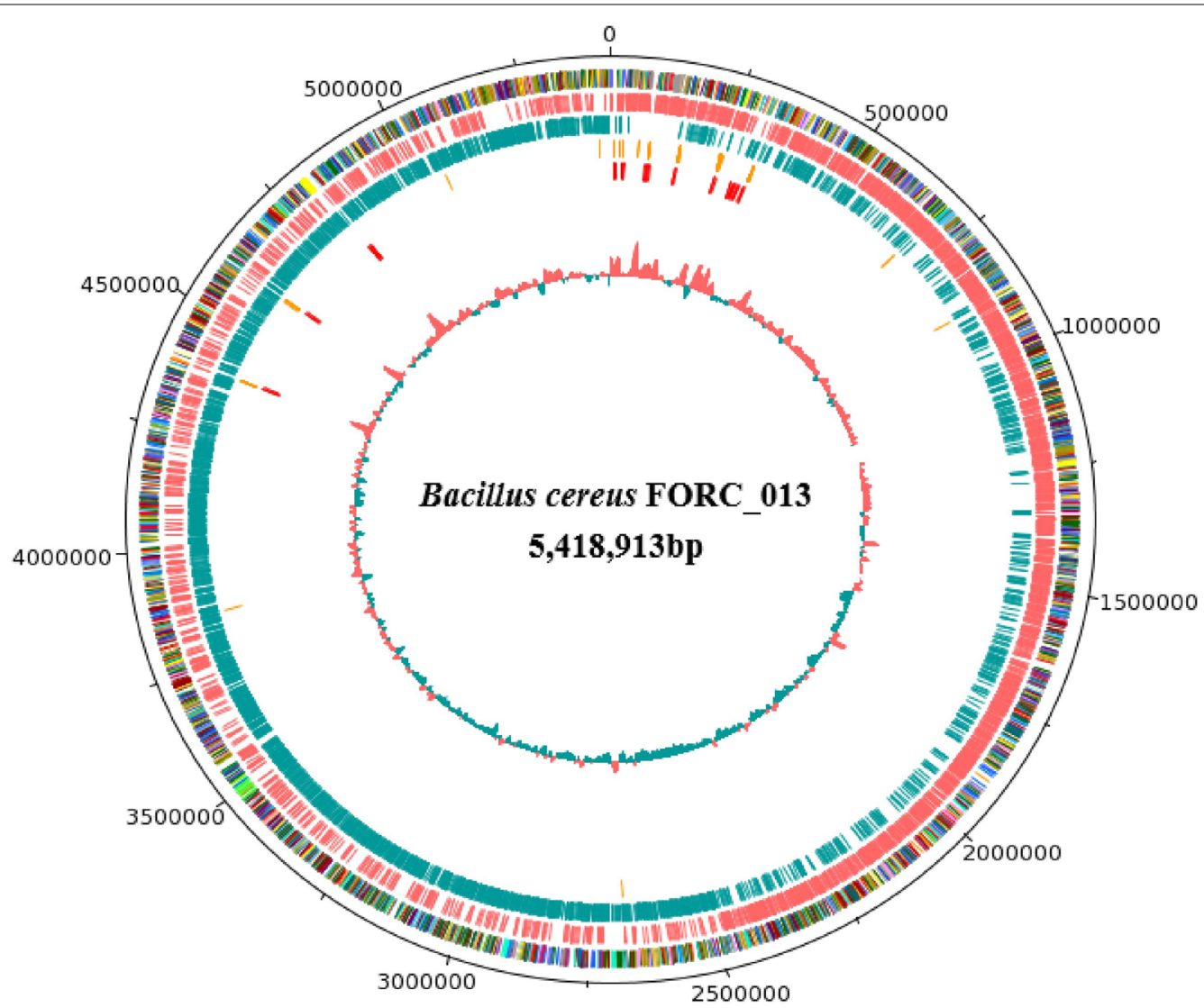

E Amino acid transport and metabolism D Cell cycle control, cell division, chromosome partitioning

A RNA processing and modification

J Translation ribosomal structure and biogenesis

- G Carbohydrate transport and metabolism

- K Transcription

H Coenzyme transport and metabolism

- R General function prediction only

V Defense mechanisms

I Lipid transport and metabolism

- T Signal transduction mechanisms

N Cell motility

U Intracellular trafficking, secretion and vesicular transport

S Function unknown

Q Q Secondary metabolites biosynthesis, transport and catabolism

- L Replication,recombination adn repair

M Cell wall/membrane/envelope biogenesis

O Posttranslational modification, protein turnover, chaperones

- C Energy production and conversion

- F Nucleotide transport and metabolism

$\operatorname{CDS}(+)$

- $\operatorname{CDS}(-)$

tRNA

P Inorganic ion transport and metabolism

Fig. 1 Circular genome map of the B. cereus FORC_013 chromosome. Circles, from outer to inner, represent the Cluster of Orthologous Groups (COG) distribution, with protein coding sequences (CDS) in the leading strand, CDS in the lagging strand, tRNA, rRNA, and the GC content. Functional genes are labeled around the outer circle as evolutionarily selected genes

database and the FORC_013 genome sequence (Fig. 3). The neighbor-joining method was used to construct an ANI tree with pairwise distance matrix and a phylogenetic tree with orthologous genes. The ATCC14579 strain was shown to contain pathogenicity-related genes in a previous study [33]. In both of our tree analyses, FORC_013 clustered closely with ATCC14579. The high ANI value (98.6\%) indicates that FORC_013 may have genes affecting virulence, similar to ATCC14579.
To identify the positively selected genes, we calculated the positive selection sites for the orthologous gene using the branch and branch-site models. In the branch model, 12 genes were revealed as being selected: YtxC-like family protein, post-transcriptional regulator ComN, cytochrome c-550, HTH-type transcriptional regulator NorG, putative HTH-type transcriptional regulator, flagellar hook-basal body complex protein FliE, putative murein peptide carboxypeptidase, HTH-type 

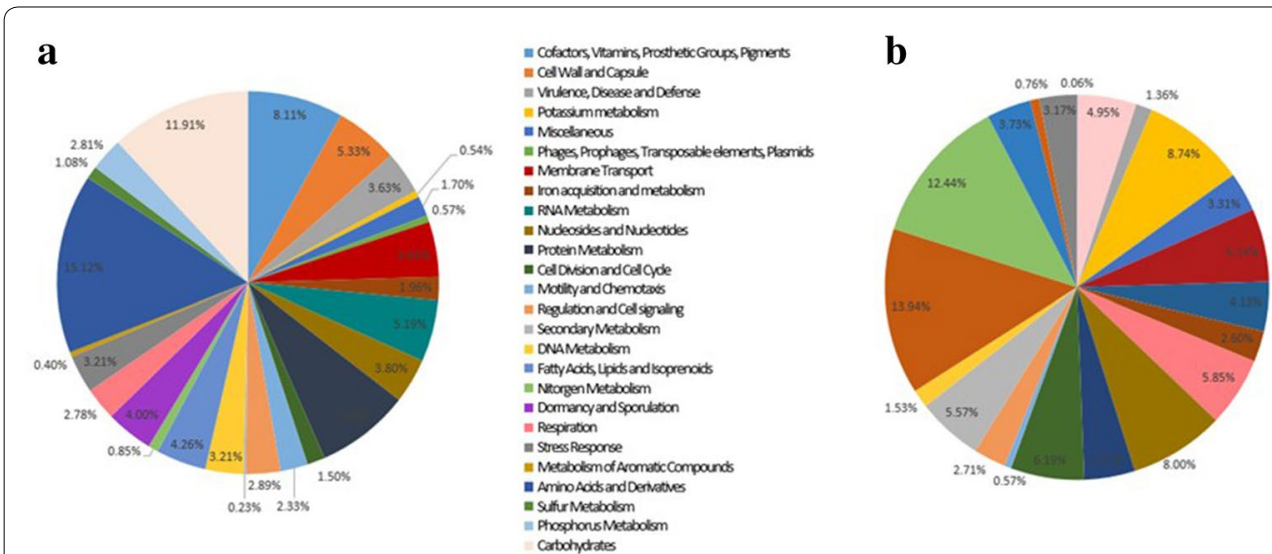

|l RNA procescing and modicotion

Energy prodution and converson

- Cel odecontod, ofl divion and dromosome partioning

II Amino acd tursoport and metabolsm

- Nudectide trasportand metabolism

- Carbohydratetansportand metaboism

- Coenesmetarsportandmetabolism

- Lipid trasoport and metabolism

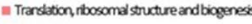

- Trascotion

- Replication recombination and repar

- Cellwal/mertirane/enelope biogenes

= Celmotity

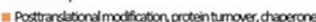

" norgricion transportand metabolem

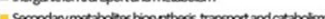

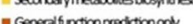

11: Function univiom

II Senat transductionmechanisms

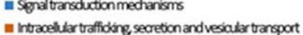

In Defersemecherions

Fig. 2 Functional categorization of all estimated open reading frames (ORFs) in the B. cereus FORC_013 genome based on the a SEED and $\mathbf{b}$ COG databases

Table 1 Virulence factors of $B$. cereus FORC_013

\begin{tabular}{lll}
\hline Virulence factor & Annotation & Locus tag \\
\hline Host immune evasion & & Folysaccharide capsule \\
- & & FORC13_5198, FORC13_5217 \\
Lipase & Phosphatidylcholine-preferring phospholipase C (PC-PLC) & FORC13_4514 \\
plcA & Phosphatidylinositol-specific phospholipase C (PI-PLC) & FORC13_1400 \\
Protease & & FORC13_4518 \\
inhA & Immune inhibitor A metalloprotease & FORC13_3892 \\
- & Immune inhibitor A metalloprotease & FORC13_5291 \\
Regulation & & FORC13_5042 \\
plcR & PlcR & FORC13_4071 \\
Toxin & & FORC13_1637 \\
- & Anthrolysin O & FORC13_3034 \\
cytK & Cytotoxin K & FORC13_5388 \\
hlylll & Hemolysin II & FORC13_2078 FORC13_2081 \\
- & Hemolysin III \\
hblC, hblD, hblB, hblA & Hemolysin III homolog \\
nheC, nheB, nheA & Hemolytic enterotoxin HBL & FORC13_3362 FORC13_3364 \\
Others & Non-hemolytic enterotoxin NHE & FORC13_4633 \\
- & Internalin-like & FORC13_3846
\end{tabular}

transcriptional regulator GltC, AT synthase subunit a, regulatory protein YeiL, superoxide dismutase $(\mathrm{Mn}) 2$, and oligoendopeptidase F (Additional file 1: Table S2). In the branch-site model, two putative genes were detected with their own functions: mycinamicin III $3^{\prime \prime}$-O-methyltransferase and putative efflux system component YknX (Additional file 1: Table S3). In the methyltransferase gene, amino acid 190 was changed to asparagine from aspartic acid in FORC_013. Asparagine and aspartic acid are categorized in the carboxamide group and the negatively charged group, respectively. In the gene identified as a putative efflux system component, amino acid 153 in FORC_013 was changed to isoleucine from valine. Isoleucine is in the hydrophobic group, while valine is categorized as non-polar. Through evolutionary analysis, we detected some positively selected genes related to virulence. The superoxide dismutase $(\mathrm{Mn}) 2$ plays a crucial role in protecting cells from the oxidative stress [34, 35]. 

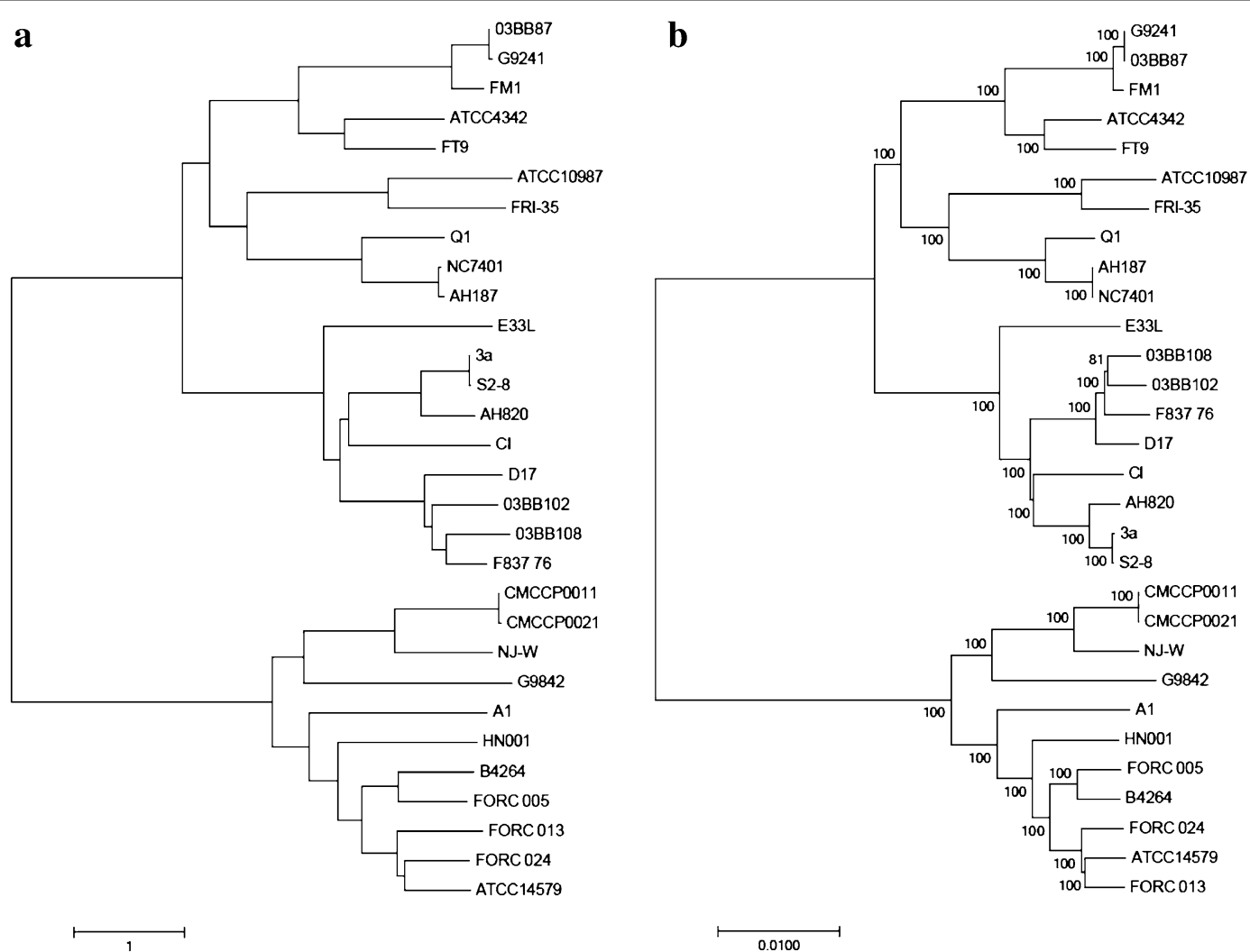

Fig. 3 Average nucleotide identity (ANI) tree and phylogenetic tree based on a ANI value and $\mathbf{b}$ orthologous genes, respectively

Toxin from FORC_013 can survive low gastric pH condition in the presence of the superoxide dismutase $(\mathrm{Mn})$ 2. The YknX gene encodes an $A B C$ transporter, which is contributed to the export of virulence factor [36, 37]. These results suggest that the positively selected genes identified in the FORC_013 strain may have an influence on pathogenicity.

Furthermore, Pan-genome analysis of 30 strains revealed 25,247 genes comprising the supra-genome based on the Roary pipeline (Additional file 1: Fig S2a). The relation between the number of genomes $(x)$ and the pan-genome size $(y)$ was $y=7520.62 x^{0.37}-2066.4$ $\left(R^{2}=0.999926\right)$. Also, the relationship between the core genome size and the genome number was calculated as $n=7276.95 \mathrm{e}^{-0.82 m}+2284.85\left(R^{2}=0.960822\right)$. The size of the $B$. cereus pan-genome has grown, while the scale of core genome has decreased with the addition of new strains (Additional file 1: Fig S2b). Based on this result, we can consider this pan-genome to be an open pan-genome, providing evidence that this species dwells under conditions that encourage the transfer of genetic material through pathways such as horizontal gene transfer [38, 39]. Above all, we examined the unique genes of FORC_013 to elucidate the strain's specific biological characteristics. We detected that the unique genes of the FORC_013 strain comprise 224 genes, including 130 hypothetical proteins (Additional file 2: Table S1). The proportion of unique genes of FORC_013 was $4.16 \%$ (Additional file 1: Fig S2c). Furthermore, we could detect strain-specific genes of FORC_013 associated with virulence through Pangenome analysis. Here, we identified A-type flagellin and flagellin genes that are involved in biofilm formation [40], which are candidates for assisting the activation of FORC_013's pathogenicity.

\section{Conclusions}

In this study, we sequenced the genome of $B$. cereus FORC_013, which is an opportunistic pathogen that occurs food-borne illness, and performed comparative analysis with 29 published strains. As a result, we detected the virulence factors of this strain that can assist its pathogenicity. We also identified positively selected genes and unique genes of FORC_013. This study advances our understanding of the genetic characteristics of FORC_013. In addition, these findings will provide useful information for further research related to the virulence mechanisms used by this pathogen. 


\section{Additional files}

Additional file 1. Additional Tables and Figures.

Additional file 2: Table S1. Additional Tables.

\section{Abbreviations}

ORFs: open reading frames; PROKKA: rapid annotation of prokaryotic genomes; RAST: rapid annotation using subsystem technology; COG: Cluster of Orthologous Groups; BLAST: basic local alignment search tool; ANI: average nucleotide identity; CDS: protein coding sequences.

\section{Authors' contributions}

$\mathrm{SR}, \mathrm{JHL}, \mathrm{SHC}$, and HK supervised this project. HJK performed the comparative genomics, analyzed the data, and wrote the manuscript. KK and SA conducted the sequence quality check, assembly, gene prediction, and gene annotation. HYC and SK conducted experiments. HJK and SA reviewed and improved the manuscript. All authors read and approved the final manuscript.

\section{Author details}

${ }^{1}$ Department of Agricultural Biotechnology and Research Institute of Agriculture and Life Sciences, Seoul National University, Seoul, Republic of Korea. ${ }^{2}$ Interdisciplinary Program in Bioinformatics, Seoul National University, Seoul, Republic of Korea. ${ }^{3}$ Food-Borne Pathogen Omics Research Center (FORC), Seoul National University, Seoul, Republic of Korea. ${ }^{4}$ Department of Agricultural Biotechnology, Center for Food Safety and Toxicology, Seoul National University, Seoul, Republic of Korea. ${ }^{5}$ Department of Food Science and Biotechnology, Institute of Life Science and Resources, Kyung Hee University, Yongin, Republic of Korea.

\section{Acknowledgements}

Not applicable.

\section{Competing interests}

The authors declare that they have no competing interests.

\section{Availability of data and materials}

Genome sequences of Bacillus cereus FORC 013 were deposited in the NCBI GenBank under the Accession Numbers CP011145.1 and CP011146.1 for chromosome and a plasmid, respectively.

\section{Funding}

This research was supported by a Grant (14162MFDS972) from Ministry of Food and Drug Safety, Korea in 2017.

\section{Publisher's Note}

Springer Nature remains neutral with regard to jurisdictional claims in published maps and institutional affiliations.

Received: 10 March 2017 Accepted: 21 April 2017

Published online: 15 May 2017

\section{References}

1. Newell DG, Koopmans M, Verhoef L, Duizer E, Aidara-Kane A, Sprong H, Opsteegh M, Langelaar M, Threfall J, Scheutz F. Food-borne diseasesthe challenges of 20 years ago still persist while new ones continue to emerge. Int J Food Microbiol. 2010;139:S3-15.

2. Scallan E, Hoekstra RM, Angulo FJ, Tauxe RV, Widdowson M-A, Roy SL, Jones JL, Griffin PM. Foodborne illness acquired in the United Statesmajor pathogens. Emerg Infect Dis. 2011;17:7-15.

3. Altekruse S, Cohen M, Swerdlow D. Emerging foodborne diseases. Emerg Infect Dis. 1997;3:285.

4. Tauxe RV. Emerging foodborne diseases: an evolving public health challenge. Emerg Infect Dis. 1997;3:425.
5. Rasko DA, Altherr MR, Han CS, Ravel J. Genomics of the Bacillus cereus group of organisms. FEMS Microbiol Rev. 2005;29:303-29.

6. Carretto E, Barbarini D, Poletti F, Capra FM, Emmi V, Marone P. Bacillus cereus fatal bacteremia and apparent association with nosocomial transmission in an intensive care unit. Scand J Infect Dis. 2000:32:98-100.

7. Kotiranta A, Lounatmaa K, Haapasalo M. Epidemiology and pathogenesis of Bacillus cereus infections. Microbes Infect. 2000;2:189-98.

8. Johnson K, Nelson C, Busta F. Germination and heat resistance of Bacillus cereus spores from strains associated with diarrheal and emetic foodborne illnesses. J Food Sci. 1982;47:1268-71.

9. Granum PE, Lund T. Bacillus cereus and its food poisoning toxins. FEMS Microbiol Lett. 1997;157:223-8.

10. Granum PE. Bacillus cereus. In: Fratamico PM, Bhunia AK, Smith JL, editors. Foodborne pathogens: microbiology and molecular biology. Norfolk: Caister Academic Press; 2005. p. 409-14.

11. Guinebretière $M-H$, Broussolle V. Enterotoxigenic profiles of foodpoisoning and food-borne Bacillus cereus strains. J Clin Microbiol. 2002:40:3053-6.

12. Chin C-S, Alexander DH, Marks P, Klammer AA, Drake J, Heiner C, Clum A, Copeland A, Huddleston J, Eichler EE. Nonhybrid, finished microbia genome assemblies from long-read SMRT sequencing data. Nat Methods. 2013;10:563-9.

13. Delcher AL, Salzberg SL, Phillippy AM. Using MUMmer to identify similar regions in large sequence sets. Curr Protoc Bioinform. 2003;10:3.1-8.

14. Hall T. BioEdit: an important software for molecular biology. GERF Bull Biosci. 2011;2:6.

15. Seemann T. Prokka: rapid prokaryotic genome annotation. Bioinformatics. 2014;18:btu153.

16. Aziz RK, Bartels D, Best AA, DeJongh M, Disz T, Edwards RA, Formsma K, Gerdes S, Glass EM, Kubal M. The RAST Server: rapid annotations using subsystems technology. BMC Genom. 2008;9:1.

17. Wu S, Zhu Z, Fu L, Niu B, Li W. WebMGA: a customizable web server for fast metagenomic sequence analysis. BMC Genom. 2011:12:1.

18. Carver T, Thomson N, Bleasby A, Berriman M, Parkhill J. DNAPlotter: circular and linear interactive genome visualization. Bioinformatics. 2009:25:119-20.

19. Goris J, Konstantinidis KT, Klappenbach JA, Coenye T, Vandamme P, Tiedje JM. DNA-DNA hybridization values and their relationship to wholegenome sequence similarities. Int J Syst Evol Microbiol. 2007;57:81-91.

20. Kim KM, Sung S, Caetano-Anollés G, Han JY, Kim H. An approach of orthology detection from homologous sequences under minimum evolution. Nucleic Acids Res. 2008:36:e110.

21. Löytynoja A, Goldman N. An algorithm for progressive multiple alignment of sequences with insertions. Proc Natl Acad Sci USA. 2005;102:10557-62

22. Talavera G, Castresana J. Improvement of phylogenies after removing divergent and ambiguously aligned blocks from protein sequence alignments. Syst Biol. 2007;56:564-77.

23. Kumar S, Stecher G, Tamura K. MEGA7: molecular evolutionary genetics analysis version 7.0 for bigger datasets. Mol Biol Evol. 2016:33:1870-4

24. Yang Z. PAML 4: phylogenetic analysis by maximum likelihood. Mol Biol Evol. 2007:24:1586-91.

25. Page AJ, Cummins CA, Hunt M, Wong VK, Reuter S, Holden MT, Fookes M, Falush D, Keane JA, Parkhill J. Roary: rapid large-scale prokaryote pan genome analysis. Bioinformatics. 2015;31:3691-3.

26. Guillemet $\mathrm{E}$, Cadot $\mathrm{C}$, Tran $\mathrm{S}-\mathrm{L}$, Guinebretière $\mathrm{M}-\mathrm{H}$, Lereclus D, Ramarao $\mathrm{N}$. The InhA metalloproteases of Bacillus cereus contribute concomitantly to virulence. J Bacteriol. 2010;192:286-94.

27. Andreeva-Kovalevskaya ZI, Solonin A, Sineva E, Ternovsky V. Pore-forming proteins and adaptation of living organisms to environmental conditions. Biochem (Mosc). 2008:73:1473-92.

28. Baida GE, Kuzmin NP. Mechanism of action of hemolysin III from Bacillus cereus. Biochim Biophys Acta (BBA) Biomembr. 1996;1284:122-4.

29. Salamitou S, Ramisse F, Brehélin M, Bourguet D, Gilois N, Gominet M, Her-

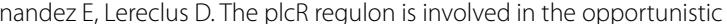
properties of Bacillus thuringiensis and Bacillus cereus in mice and insects. Microbiology. 2000;146:2825-32.

30. Hsueh Y-H, Somers EB, Lereclus D, Wong ACL. Biofilm formation by Bacillus cereus is influenced by $\mathrm{PlcR}$, a pleiotropic regulator. Appl Environ Microbiol. 2006:72:5089-92. 
31. Ryu J-H, Beuchat LR. Biofilm formation and sporulation by Bacillus cereus on a stainless steel surface and subsequent resistance of vegetative cells and spores to chlorine, chlorine dioxide, and a peroxyacetic acid-based sanitizer. J Food Prot ${ }^{\circledR}$. 2005;68:2614-22.

32. Majed R, Faille C, Kallassy M, Gohar M. Bacillus cereus biofilms—same only different. Front Microbiol. 2016;7:1054.

33. Ivanova N, Sorokin A, Anderson I, Galleron N, Candelon B, Kapatral V, Bhattacharyya A, Reznik G, Mikhailova N, Lapidus A. Genome sequence of Bacillus cereus and comparative analysis with Bacillus anthracis. Nature. 2003;423:87-91.

34. Wang Y, Mo X, Zhang L, Wang Q. Four superoxide dismutase (isozymes) genes of Bacillus cereus. Ann Microbiol. 2011;61:355-60.

35. Duport C, Jobin M, Schmitt P. Adaptation in Bacillus cereus: from stress to disease. Front Microbiol. 2016;7:1550.
36. Butcher BG, Helmann JD. Identification of Bacillus subtilis $\sigma W$-dependent genes that provide intrinsic resistance to antimicrobial compounds produced by Bacilli. Mol Microbiol. 2006;60:765-82.

37. Davidson AL, Dassa E, Orelle C, Chen J. Structure, function, and evolution of bacterial ATP-binding cassette systems. Microbiol Mol Biol Rev. 2008;72:317-64.

38. Polz MF, Alm EJ, Hanage WP. Horizontal gene transfer and the evolution of bacterial and archaeal population structure. Trends Genet. 2013;29:170-5.

39. Muzzi A, Donati C. Population genetics and evolution of the pan-genome of Streptococcus pneumoniae. Int J Med Microbiol. 2011;301:619-22.

40. Houry A, Briandet R, Aymerich S, Gohar M. Involvement of motility and flagella in Bacillus cereus biofilm formation. Microbiology. 2010;156:1009-18.

\section{Submit your next manuscript to BioMed Central and we will help you at every step:}

- We accept pre-submission inquiries

- Our selector tool helps you to find the most relevant journal

- We provide round the clock customer support

- Convenient online submission

- Thorough peer review

- Inclusion in PubMed and all major indexing services

- Maximum visibility for your research

Submit your manuscript at www.biomedcentral com/submit 